\title{
Multi-objective Optimization of Orbit Transfer Trajectory Using Imperialist Competitive Algorithm
}

\author{
Abolfazl Shirazi \\ BCAM - Basque Center for Applied Mathematics \\ Mazarredo, 14 \\ 48009 Bilbao, Basque Country, Spain \\ (+34) 946567842 \\ ashirazi@bcamath.org
}

\begin{abstract}
This paper proposes a systematic direct approach to carry out effective multi-objective optimization of space orbit transfer with high-level thrust acceleration. The objective is to provide a transfer trajectory with acceptable accuracy in all orbital parameters while minimizing spacecraft fuel consumption.

With direct control parameterization, in which the steering angles of thrust vector are interpolated through a finite number of nodes, the optimal control problem is converted into the parameter optimization problem to be solved by nonlinear programming. Besides the thrust vector direction angles, the thrust magnitude is also considered as variable and unknown along with initial conditions. Since the deviation of thrust vector in spacecraft is limited in reality, mathematical modeling of thrust vector direction is carried out in order to satisfy constraints in maximum deviation of thrust vector direction angles. In this modeling, the polynomial function of each steering angle is defined by interpolation of a curve based on finite number of points in a specific range with a nominal center point with uniform distribution. This kind of definition involves additional parameters to the optimization problem which results the capability of search method in satisfying constraint on the variation of thrust direction angles.
\end{abstract}

Thrust profile is also modeled based on polynomial functions of time with respect to solid and liquid propellant rockets. Imperialist competitive algorithm is used in order to find optimal coefficients of polynomial for thrust vector and the optimal initial states within the transfer. Results are mainly affected by the degree of polynomials involved in mathematical modeling of steering angles and thrust profile which results different optimal initial states where the transfer begins. It is shown that the proposed method is fairly beneficial in the viewpoint of optimality and convergence. The optimality of the technique is shown by comparing the finite thrust optimization with the impulsive analysis.

Comparison shows that the accuracy is acceptable with respect to fair precision in orbital elements and minimum fuel mass. Also, the convergence of the optimization algorithm is investigated by comparing the solution of the problem with other optimization techniques such as Genetic Algorithm. Results confirms the practicality of Imperialist Competitive Algorithm in finding optimum variation of thrust vector which results best transfer accuracy along with minimizing fuel consumption.

\section{TABLE OF CONTENTS}

1. INTRODUCTION 1

2. STATEMENT OF THE PROBLEM.................................. 2
3. OPTIMIZATION MODEL OF HIGH-THRUST TRAJECTORIES

4. TRAJECTORY DESIGN BASED ON DIRECT

STRATEGY 4

5. IMPERIALIST COMPETITIVE ALGORITHM

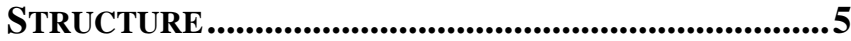

6. SIMULATION..............................................................6

7. CONCLUSION AND DISCUSSION ..............................12

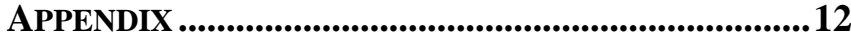

ACKNOWLEDGEMENTS ......................................13

REFERENCES .......................................................13

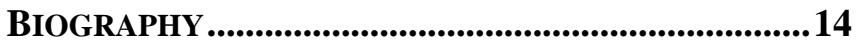

\section{INTRODUCTION}

Most successful trajectory optimization methods are gradient-based, mainly due to their computational efficiency. However, these algorithms are local in nature and it is easy for the solution to get trapped in a local minimum. To avoid this, global rather than local search must be performed. In recent years, interest in the application of evolutionary algorithms (EAs) to trajectory optimization has grown, substantially due to their global search capabilities.

In [1] Genetic Algorithm (GA) is used by Taheri and Abdelkhalik to generate initial trajectories in a three-body dynamical framework assuming the use of a low-thrust propulsion system. The GA can also be employed to optimize the rendezvous trajectory for asteroid deflection missions [2], or used to control of asteroid retrieval trajectories to libration point orbits [3].

Besides GA, Particle Swarm Optimization (PSO), an evolutionary algorithm based on observation and simulation of the social behavior of flocks of birds, has been widely used in space orbit trajectory design. Dileep et al. [4] used PSO in ascent phase launch vehicle trajectory optimization problem. Alonso Zotes and Santos Penas [5] employed PSO in designing an interplanetary trajectory for Earth to Jupiter and Saturn missions.

A recently introduced evolutionary meta-heuristic which has not received much attention in the aerospace community is the Imperialist Competitive Algorithm (ICA) [6], which is a global optimization technique based on the behavior of imperialists in their attempt to conquer colonies. The algorithm is simple and can be implemented in a few lines 
of computer code. Moreover, it is gradient-free and can solve irregular optimization problems. These characters make ICA an easy-to-use algorithm for real-life problems such as the trajectory optimization, and multidisciplinary design optimization (MDO).

This algorithm has been applied in optimization problems in many engineering researches such as controller design [7], logic circuit optimization [8], power flow optimization [9] and virtual machine placement [10]. The only fair application of ICA in trajectory optimization of spacecraft has been presented by Shafieenejad et al. [11] in which the optimal control problems in a low-thrust space orbit transfer problem is tackled using ICA with regard to path design viewpoint and free initial condition.

In this work, ICA is employed to find the optimum transfer trajectory for spacecraft based on using high-thrust propulsion systems. The use of ICA in high-thrust orbital maneuvers can be considered as a novel approach in trajectory optimization since this algorithm hasn't been previously employed in such orbit transfers.

The rest of this paper is organized as follows. Statement of the problem is given in Section 2. Section 3 presents the high-thrust dynamic model, various performance indexes, and boundary constraints. Section 4 describes the optimization approach of high-thrust orbit transfers. Structure of Imperialist Competitive Algorithm for design of high-thrust trajectories is defined in Section 5. In Section 6 , the simulation case of optimal transfer trajectories with boundary constraints is studied to validate the general rapid design and optimization framework developed in this paper. Finally, Section 7 contains conclusion and future work.

\section{STATEMENT OF THE PROBLEM}

Consider a general orbit transfer from initial orbit $\left(a_{i}, e_{i}, i_{i}, \omega_{i}, \Omega_{i}\right)$ to final orbit $\left(a_{f}, e_{f}, i_{f}, \omega_{f}, \Omega_{f}\right)$ as depicted in Figure 1.

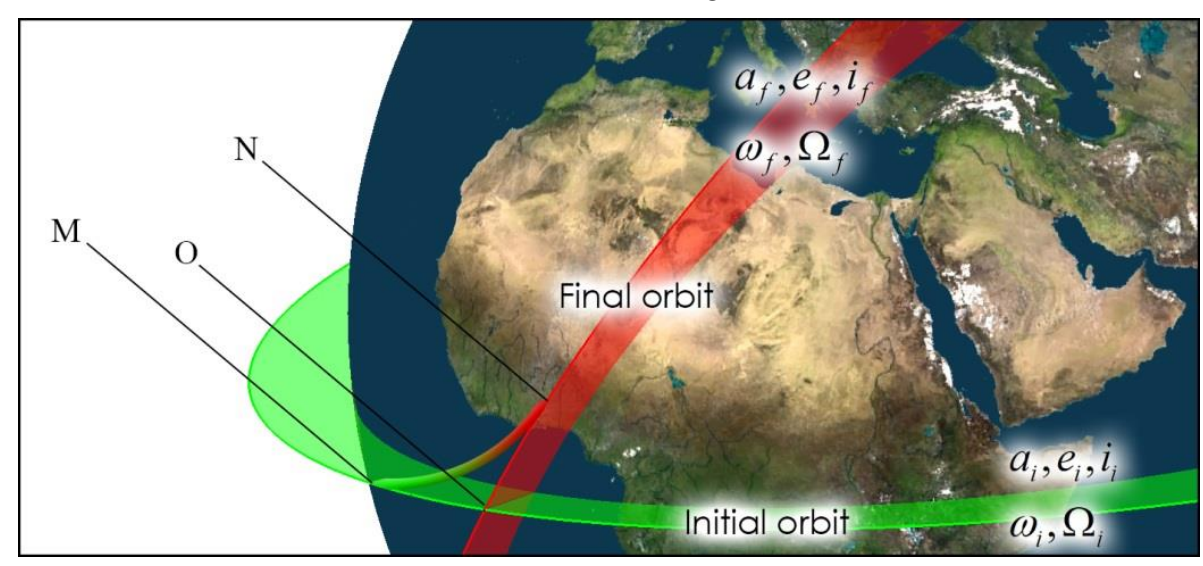

Figure 1. Schematic view of trajectories in finite-thrust orbital maneuver

Based on impulsive assumption, orbit transfer occurs at the intersection of initial and final orbits (O). However, regarding finite-thrust assumption in which the transfer time is not equal to zero, spacecraft motion will be on a non-Keplerian trajectory known as burn arc during orbit transfer. The burn arc starts and ends in unknown points on initial and final orbits respectively $(\mathrm{M}, \mathrm{N})$. While the spacecraft moves on burn arc, orbital parameters have continuous changes. The orbit transfer will be optimal if the orbital parameters at the end of transfer are equal or near equal to desired values while the spacecraft have minimum fuel consumption. In order to achieve optimal transfer, variation of thrust vector needs to be specified so that the orbital parameters reach the desired values related to final orbit while minimizing the fuel mass. The optimization technique in this article is applied to space missions where two orbits intersect at a point. However, if two orbits don't have any intersections, the space mission can simply be divided into two orbit transfers using Hohmann transfer approach [12] or any other transfer orbit which have intersections with both initial and final orbits.

\section{OPTIMIZATION MODEL OF HIGH-THRUST TRAJECTORIES}

\section{High-thrust dynamic model}

The high-thrust spacecraft is mainly affected by the gravitation of the center celestial body and engine thrust during transfer phase. For convenience, all perturbation effects are intentionally ignored since the burning time is short and the acceleration of the engine is too much in comparison to orbital perturbations, and then the equations of motion of the spacecraft with a high-thrust engine in the Cartesian coordinate can be formulated as follows [13]:

$$
\begin{gathered}
\overrightarrow{\dot{r}}=\vec{v} \\
\overrightarrow{\dot{v}}=-\frac{\mu}{r^{3}} \vec{r}+\frac{T}{m} \vec{u} \\
\dot{m}=-\frac{T}{I_{s p} g_{0}}
\end{gathered}
$$


where $\vec{r}$ and $\vec{v}$ are the position and velocity vector of the high-thrust spacecraft respectively. $m$ denotes the mass of spacecraft. $\mu$ is the gravitational parameter of the central attracting body. $\vec{u}$ represents the thrust acceleration direction, which is defined as a unit vector. $T$ stands for the thrust magnitude. $g$ is the Earth sealevel gravitational acceleration. $I_{s p}$ denotes the specific impulse of a high-thrust engine.

\section{Performance indexes and boundary constraints}

The problem of high-thrust trajectory optimization is essentially an optimal control problem, where the control variables are the thrust direction vector, and the state variables are the velocity, position and mass of the spacecraft. Unlike low-thrust transfers, the objective function of high-thrust trajectory optimization depends on the detailed mission requirements, and the common objective functions usually include the form of fuel consumption and transfer accuracy.

The problem of high-thrust trajectory optimization can be defined as searching for the appropriate thrust direction such that the fuel consumption and the transfer accuracy is optimal in a specific sense. At the same time, the constraints of equations of motion, ephemeris and maximum value of thrust magnitude and maximum thrust deviation should be not violated.

In order to optimize a transfer trajectory, a scalar cost function must be defined to measure the trajectory's merit. Since the transfer is not coplanar in general, both the shape and orientation of final orbit is considered as the target in optimization. Therefore, the goal is to maximize the accuracy of final orbits in this transfer with respect to provided thrust profile. Considering $t_{f}$ as the total burn time in orbit transfer, the cost function representing the transfer accuracy may be defined as [14]:

$$
\begin{aligned}
J_{e} & =\left(\frac{a\left(t_{f}\right)-a_{\text {des }}}{\sigma_{a}}\right)^{2}+\left(\frac{e\left(t_{f}\right)-e_{\text {des }}}{\sigma_{e}}\right)^{2} \\
& +\left(\frac{i\left(t_{f}\right)-i_{\text {des }}}{\sigma_{i}}\right)^{2}+\left(\frac{\omega\left(t_{f}\right)-\omega_{\text {des }}}{\sigma_{\omega}}\right)^{2} \\
& +\left(\frac{\Omega\left(t_{f}\right)-\Omega_{\text {des }}}{\sigma_{\Omega}}\right)^{2}
\end{aligned}
$$

where $a, e, i, \omega$ and $\Omega$ denote the semi-major axis, eccentricity, argument of perigee and right ascension of ascending node at the end of orbit transfer respectively. Similarly, $a_{d e s}, e_{d e s}, i_{d e s}, \omega_{d e s}$ and $\Omega_{d e s}$ represent the desired values of related parameters at the end of orbit transfer. Also, $\sigma_{a}, \sigma_{e}, \sigma_{i}, \sigma_{\omega}$ and $\sigma_{\Omega}$ are weighting coefficients related to each parameters which are specified based on desired accuracy according to mission objective.
Derivation of orbital parameters in Eq. (4) from state variables $(\vec{r}, \vec{v})$ is provided in Appendix.

This definition of cost function fairly scales the weighting coefficients. As based on this definition, if $J_{e}<1$, all of the parameters at the end of orbit transfer reach the desired values with acceptable accuracies.

Besides $J_{e}$ which represents the orbit accuracy, another cost function need to be specified representing the fuel consumption of the spacecraft as below:

$$
J_{m}=\left(\frac{m_{f}-m_{i m}}{\sigma_{m}}\right)^{2}
$$

where $m_{f}$ represents the actual required fuel mass for the transfer in finite thrust simulation while $\sigma_{m}$ is the weighting coefficient of fuel mass. Considering $m_{i m}$ as the required fuel mass regarding impulsive analysis, the inequality $m_{f}>m_{i m}$ holds since the fuel mass calculated by the impulsive assumption is the least fuel mass that is required for a space mission in reality. However, since there is always an acceptable radius of error in the final orbital elements defined by weighting coefficients in Eq. $5, \sigma_{m}$ can be considered equal to $m_{i m}$ as a proper choice for every mission objective.

Similarly, if $J_{m}<1$, the fuel mass of the spacecraft will be less than the expected value defined by $\sigma_{m}$.

Having the cost functions for transfer accuracy $\left(J_{e}\right)$ and fuel consumption $\left(J_{m}\right)$, the overall cost function can be defined as below:

$$
J=\frac{1}{2}\left(\lambda J_{e}+(1 / \lambda) J_{m}\right)
$$

where $\lambda$ is the overall scale factor with boundary of $0<\lambda$ which represents the balance between fuel optimality and orbit accuracy. The scale factor is specified based on the desirability of two criteria.

In overall, regarding the above definitions, the multiobjective optimization problem turns into single objective one regarding weighting coefficients and scale factor. The stopping criterion of the optimization process would be $J<1$ since the two minor cost functions have been already scaled using weighting coefficients. The main problem which is tackled in this research is minimizing the overall cost function defined as Eq. 6 by finding optimal thrust vector direction and optimal thrust magnitude profile as functions of time along with optimal starting position of spacecraft on initial orbit. 


\section{TRAJECTORY DESIGN BASED ON DIRECT STRATEGY}

\section{Mathematical modeling of thrust vector}

The variation of thrust direction angles and thrust magnitude are to be specified so that the performance index defined by Eq. (6) becomes minimal. Considering the thrust acceleration direction as $\vec{u}=\left[\begin{array}{lll}\cos (\alpha) \cos (\beta) & \cos (\alpha) \sin (\beta) & \sin (\alpha)\end{array}\right]$, the guidance commands $(\alpha, \beta)$ along with thrust magnitude $(T)$ are initially assumed as the polynomial functions of time as:

$$
\begin{aligned}
& \alpha(t)=\sum_{i=0}^{n} a_{i} t^{i} \\
& \beta(t)=\sum_{i=0}^{n} b_{i} t^{i} \\
& T(t)=\sum_{i=0}^{n} c_{i} t^{i}
\end{aligned}
$$

where $t$ represents the time that starts with the ignition of engine rocket, $a_{i}, b_{i}$ and $c_{i}$ are the polynomial coefficients of direction angles and thrust magnitude respectively and $n$ is the degree of polynomials.

Since the thrust vector is given by the polynomial functions of time in the form of Eq. (7), (8) and (9), there is no limitation in their variation in general form. Therefore, finding optimal $a_{i}, b_{i}$ and $c_{i}$ will result large variations of direction angles and thrust magnitude. However, the deviation of thrust vector along with the magnitude in spacecraft is limited in reality. Most solid rocket engines can have steering deviation of $\pm 20^{\circ}$ at most during the burn time [15]. This maximum value has been assumed to be up to $\pm 10^{\circ}$ in some researches involving thrust vector controllers [16]. Similarly, the time profile of thrust magnitude is also a restricted function of time which is affected by the core shape of the propellant, rocket inhibitors and grain geometry [17, 18]. However, the design process of the propulsion system which satisfies a predefined thrust magnitude profile is a challenging issue and its possibility is totally depends on the available technology. Therefore; it is required to redefine the unknown parameters in optimization with respect to these limitations.

In order to apply the range constraint of steering angles and thrust magnitude in optimization process, the concept of optimization in orbit transfer problem is modified in a way so that the boundary of $\alpha, \beta$ and $T$ can be specified.

The polynomial function of each unknown function $(\alpha$, $\beta$ and $T$ ), hereinafter referred as $\delta(t)$, is defined by interpolation of a curve with degree of $n$ based on $m$ number of points $\left(p_{1}^{\delta}, p_{2}^{\delta} \ldots p_{m}^{\delta}\right)$ in range of $R_{\delta}$ with center of a nominal point $\delta_{c}$ with uniform distribution. This kind of definition involves additional parameters to the optimization problem which results the capability of search method in satisfying constraint on the variation of thrust direction angles and thrust magnitude.

Interpolation of a $n t h$ degree polynomial of $\delta(t)$ is desired in the following form:

$$
\delta(t)=\kappa_{0}+\kappa_{1} t+\ldots+\kappa_{n} t^{n}
$$

Where $\kappa_{i}$ represent unknown polynomial coefficients ( $a_{i}, b_{i}$ and $c_{i}$ ). Knowing the $m$ number of points ( $\left.p_{1}^{\delta}, p_{2}^{\delta} \ldots . p_{m}^{\delta}\right)$ in uniform distribution of time steps ( $\left.t_{1}, t_{2} \ldots . t_{m}\right)$, the residual $\left(R^{2}\right)$ is given by:

$$
R^{2}=\sum_{i=1}^{m}\left[p_{i}^{\delta}-\left(\kappa_{0}+\kappa_{1} t_{1}+\ldots+\kappa_{n} t_{i}^{n}\right)\right]^{2}
$$

The partial derivatives are:

$$
\frac{\partial\left(R^{2}\right)}{\partial \kappa_{n}}=-2 \sum_{i=1}^{m}\left[p_{i}^{\delta}-\left(\kappa_{0}+\kappa_{1} t_{1}+\ldots+\kappa_{n} t_{i}^{n}\right)\right]^{2} t_{i}^{n}
$$

Setting residual equations equal to zero leads to the equations:

$$
\kappa_{0} \sum_{i=1}^{m} t_{i}^{n}+\kappa_{1} \sum_{i=1}^{m} t_{i}^{n+1}+\ldots+\kappa_{n} \sum_{i=1}^{m} t_{i}^{2 n}=\sum_{i=1}^{m} t_{i}^{n} p_{i}^{\delta}
$$

Rewriting the equations in the matrix form yields:

$$
\left[\begin{array}{cccc}
m & \sum_{i=1}^{m} t_{i} & \ldots & \sum_{i=1}^{m} t_{i}^{n} \\
\sum_{i=1}^{m} t_{i} & \sum_{i=1}^{m} t_{i}^{2} & \ldots & \sum_{i=1}^{m} t_{i}^{n+1} \\
\ldots & \ldots & \ldots & \ldots \\
\sum_{i=1}^{m} t_{i}^{n} & \sum_{i=1}^{m} t_{i}^{n+1} & \ldots & \sum_{i=1}^{m} t_{i}^{2 n}
\end{array}\right]\left[\begin{array}{c}
\kappa_{0} \\
\kappa_{1} \\
\ldots \\
\kappa_{n}
\end{array}\right]=\left[\begin{array}{c}
\sum_{i=1}^{m} p_{i}^{\delta} \\
\sum_{i=1}^{m} t_{i} p_{i}^{\delta} \\
\ldots \\
\sum_{i=1}^{m} t_{i}^{n} p_{i}^{\delta}
\end{array}\right]
$$

In matrix notation, the matric form of equations for a polynomial fit can be reformed to:

$$
Y=X[\kappa]
$$

This can be solved by pre-multiplying by the transpose $X^{T}$.

$$
X^{T} Y=X^{T} X[\kappa]
$$

This matrix equation can be solved numerically, or can be inverted directly if it is well formed, to yield the unknown polynomial coefficients: 


$$
[\kappa]=\left(X^{T} X\right)^{-1} X^{T} Y
$$

Regarding the proposed definitions, the optimization algorithm will find the optimal center point $\delta_{c}$ plus a set of points $p_{m}^{\delta}$ in the range of $R_{\delta}$ relative to $\delta_{c}$ for each input $(\alpha, \beta$ and $T)$ instead of finding optimal polynomial coefficients of $a_{i}, b_{i}$ and $c_{i}$.

\section{Optimization approach}

Once the system of differential equations is solved up, orbital parameters as well as the overall performance index $(J)$ will be revealed at the end of orbit transfer. Schematic view of system performance in high-thrust orbit transfer is depicted in Figure 2.

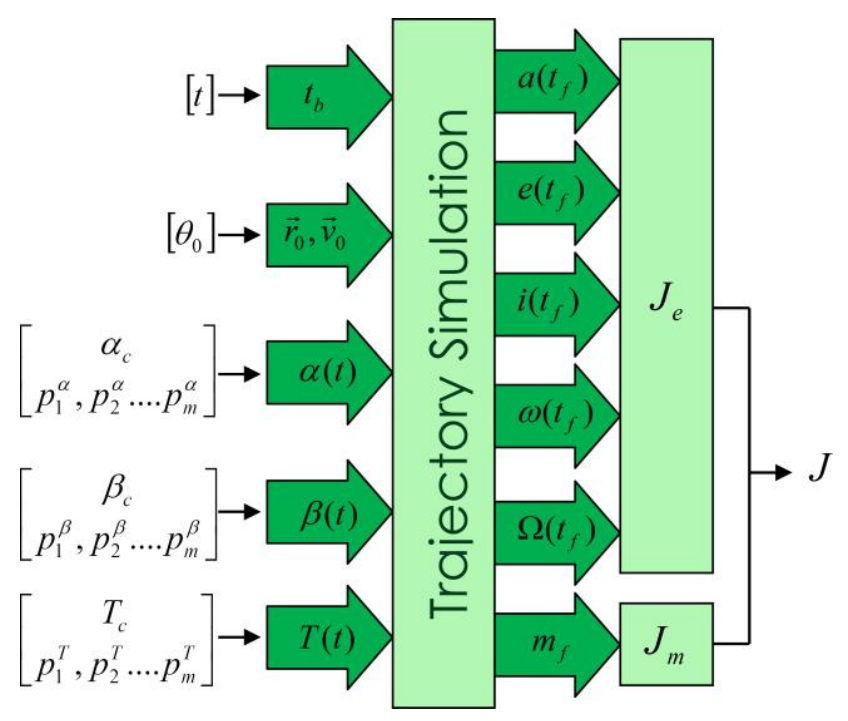

Figure 2. Diagram of cost function evaluation

Figure 2 shows system dynamics of the orbit transfer problem. Input parameters are initial states $\left(\vec{r}_{0}, \vec{v}_{0}\right)$, steering functions $(\alpha, \beta)$, thrust magnitude $(T)$ and the total burn time $\left(t_{b}\right)$. The optimal values of these parameters that results the best transfer accuracy will be found using the evolutionary algorithm.

The initial states are related to each other since the initial orbit is known. Therefore, initial true anomaly $\left(\theta_{0}\right)$ where the engine starts is considered instead of $\vec{r}_{0}, \vec{v}_{0}$. Specifying this parameter yields all six initial states. For steering angles, the nominal center points $\left(\alpha_{c}, \beta_{c}\right)$ along with several numbers of points $\left(p_{1}^{\alpha}, p_{2}^{\alpha} \ldots p_{m}^{\alpha}\right.$ and $\left.p_{1}^{\beta}, p_{2}^{\beta} \ldots p_{m}^{\beta}\right)$ subject to the constraint range of $R_{\alpha}$ and $R_{\beta}$ relative to $\alpha_{c}$ and $\beta_{c}$ are considered as described previously. The total of $2 n$ points is considered for generating the polynomial functions with degree of $n$ in current simulation. Therefore, we have $m=2 n$ in specifying polynomials. Same approach is considered for thrust magnitude which is defined based on a center point $\left(T_{c}\right)$ and selective points $\left(p_{1}^{T}, p_{2}^{T} \ldots p_{m}^{T}\right)$ and allowable range $\left(R_{T}\right)$. However, the degree of polynomials in defining thrust profile is not the same as direction angles since the variation of thrust magnitude is more restricted. Based on different thrust profiles in researches [19] including neutral, regressive and progressive thrust profiles [20], linear and cubic functions can also be considered for this variable in order to have producible thrust profile at the end of optimization. Regarding this method, the actual optimal control problem will turn into a parameter optimization problem which can be solved using ICA.

\section{IMPERIALIST COMPETITIVe Algorithm STRUCTURE}

Imperialist Competitive Algorithm (ICA), is an evolutionary algorithm that mimics the competition between imperialist countries to control more colonies in order to strengthen their empires through a process of imperialistic competition.

The ICA process is similar to other evolutionary algorithms in that it begins with an initial population, which with the ICA consists of countries. These countries are then divided into two categories: imperialists and colonies. To generate empires, the colonies are distributed among the imperialists based on their relative strength, as determined by a pre-defined criterion. The empires then compete with each other to control more colonies and expand their power. As this competition loops, stronger empires expand their power by taking possession of weak colonies from weaker empires. This process is repeated until a pre-defined stopping criterion is satisfied. A detailed description of the steps involved in this algorithm is presented in the subsection below [21].

The initiation of empires starts with the creation of several arrays that contain different problem variables $\left(P_{i}\right.$

). These arrays are called "countries" in the ICA terminology. Countries are analogous to individuals in the GA. Any country can be defined as a 1 x number of variables $\left(N_{\mathrm{var}}\right)$ array, which is used for cost function evaluation. A country can be either an imperialist or a colony.

$$
\begin{aligned}
\text { Country } & =\left[p_{1}, p_{2}, \ldots, p_{N \text { var }}\right] \\
\text { Cost } & =f(\text { Country })
\end{aligned}
$$

Next, an initial population that consists of both imperialists $N_{i m p r}$ and colonies $N_{c o l n}$ is generated to form the total population $N_{p o p}$. The formation of initial empires starts by the assignment of colonies to the imperialists, based on the imperialists' relative power. The number of 
colonies an imperialist acquires is also directionally proportional to its power. This is achieved by normalizing the cost of each imperialist $\left(C_{n}\right)$ and then dividing it by the total normalized cost of all the imperialists $\left(P_{n}\right)$.

$$
\begin{gathered}
C_{n}=c_{n}-\max \left\{c_{i}\right\} \\
p_{n}=\left|\frac{C_{n}}{\sum_{i=1}^{N_{\text {inpr }}} C_{i}}\right|
\end{gathered}
$$

After the determination of the imperialists' power, colonies are randomly distributed among imperialists to create empires. The number of colonies held by each imperialist $\left(N . C_{n}\right)$ is determined as follows:

$$
N . C_{n}=\operatorname{round}\left\{p_{n} \cdot N_{c o l n}\right\}
$$

By the end of this process, several empires have been created with their relative imperialists and colonies.

After the creation of empires, colonies are moved toward their relative imperialists. If the position of a new colony happens to yield a lower cost function, then the colony exchanges its position with its imperialist. This placeexchange process is carried out for all other colonies and their imperialists as well. Then, the total power of each empire, which is the summation of its imperialist and colonial power, is evaluated in order to rank empires based on their lowest cost functions. We note that an empire's power is mainly affected by the power of its imperialist, and that the powers of colonies are very low to almost negligible.

$$
\begin{aligned}
& \text { T.C } C_{n}=\operatorname{Cost}(\text { imperialist })_{n}+ \\
& \xi \text { Mean }\left(\operatorname{Cost}(\text { colonies of empire })_{n}\right)
\end{aligned}
$$

where T.C $C_{n}$ is the total cost of an empire and $\xi$ is a value less than 1 . The usage of $\xi$ is to assign less importance to the colonies' cost and makes the empire's cost mainly dependent on the cost of its imperialist.
Next, the empires are ready to participate in the main ICA process imperialist competition. In this process, the empires compete to take possession of the weakest colonies that belong to the weakest empires. The competition is initiated by assigning a possession probability to each empire. The possession probability increases as an empire's power increases (lowest cost). To perform this process, first, a normalized total cost (N.T.C. ) is evaluated for each empire. Then, each empire's total normalized cost $\left(\right.$ N.T.C $\left.C_{n}\right)$ is divided by the sum of the total normalized cost of all the empires to obtain the possession probability for each empire $\left(P_{p n}\right)$.

$$
\begin{aligned}
\text { N.T. } C_{n} & =\text { T. } C_{n}-\max \left(T . C_{i}\right) \\
P_{p n} & =\left|\frac{N . T \cdot C_{n}}{\sum_{i=1}^{N_{i m p r}} \text { N.T.C }}\right|
\end{aligned}
$$

After evaluating the possession probability of each empire, three vectors are formed as follows:

$$
\begin{gathered}
P=\left[p_{p 1}, p_{p 2}, \ldots, p_{p n}\right] \\
R=\left[r_{1}, r_{2}, \ldots, r_{\text {Nimpr }}\right] \\
D=P-R=\left[D_{1}, \ldots, D_{n}\right]
\end{gathered}
$$

The weakest colony is then given to the empire with the maximum $D$ index. Further details are provided in [22]. The competitive process will repeat and iterate until meeting a predetermined stopping criterion or until only one empire exists.

\section{Simulation}

Consider an elliptical orbit with semi-major axis of 20000 $\mathrm{km}$ and eccentricity of 0.1 inclined by $20^{\circ}$ as the initial orbit with argument of perigee of $40^{\circ}$ and right ascension of $60^{\circ}$. The objective of space mission is to deliver its payload from initial orbit to another orbit as depicted in Figure 3. 


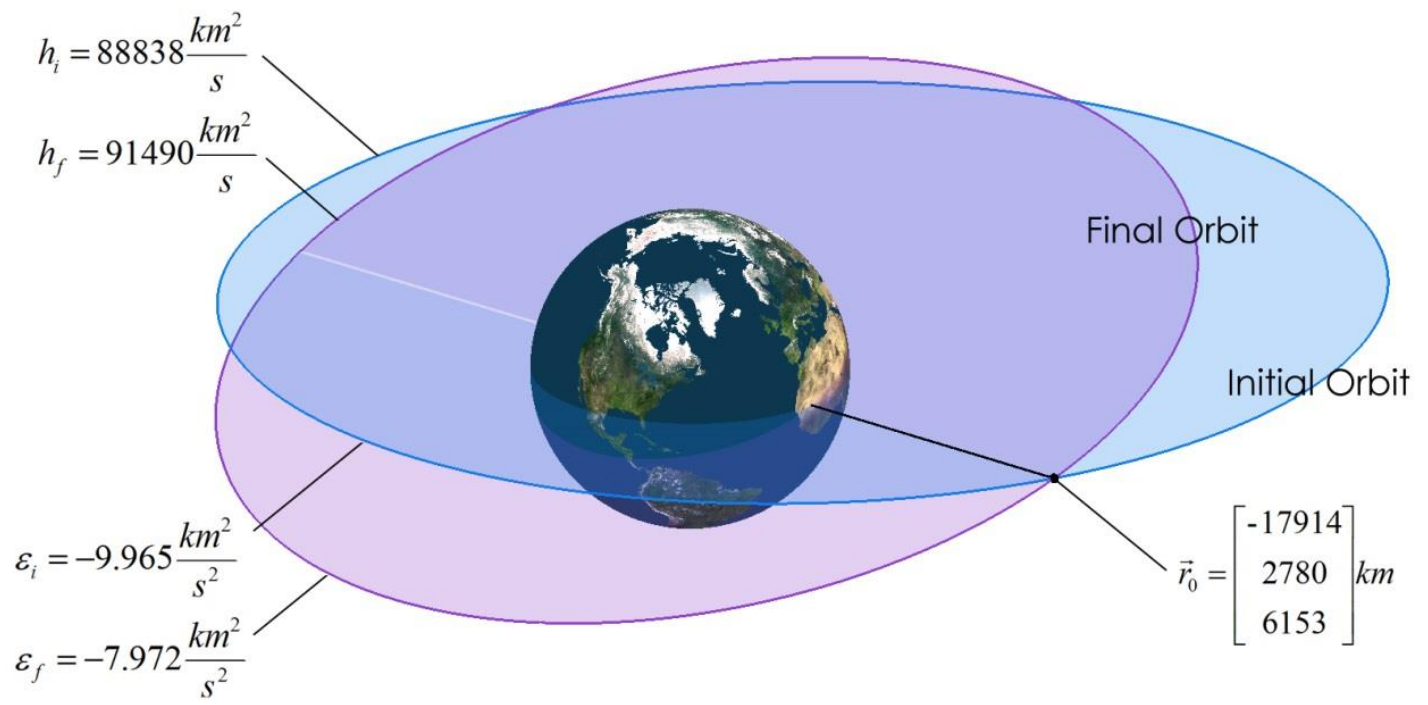

Figure 3. Three-dimensional view of sample orbit transfer

This example represents a practical problem of trajectory optimization in high-thrust transfers in which all of the orbital elements will change during the orbital maneuver.

Table 1 contains orbits characteristics in this space mission.

Table 1. Orbital parameters of initial and final trajectories

\begin{tabular}{|c|c|c|}
\hline Parameter & Initial Orbit & Final Orbit \\
\hline Eccentricity $(e)$ & 0.1 & 0.4 \\
\hline Semi-major axis $(a)$ & $20000 \mathrm{~km}$ & $25000 \mathrm{~km}$ \\
\hline $\operatorname{Inclination}(i)$ & $20^{\circ}$ & $30^{\circ}$ \\
\hline $\begin{array}{l}\text { Argument of perigee } \\
\qquad(\omega)\end{array}$ & $40^{\circ}$ & $64^{\circ}$ \\
\hline $\begin{array}{l}\text { Right ascension of } \\
\text { ascending node }(\Omega)\end{array}$ & $60^{\circ}$ & $27.2^{\circ}$ \\
\hline $\begin{array}{l}\text { Angular Momentum } \\
\qquad(h)\end{array}$ & $88838 \mathrm{~km}^{2} / \mathrm{s}$ & $\begin{array}{l}91490 \\
\mathrm{~km}^{2} / \mathrm{s}\end{array}$ \\
\hline Specific energy $(\varepsilon)$ & $-9.965 \mathrm{~km}^{2} / \mathrm{s}^{2}$ & $\begin{array}{l}-7.972 \\
\mathrm{~km}^{2} / \mathrm{s}^{2} \\
\end{array}$ \\
\hline
\end{tabular}

The expected accuracies are as follows: Acceptable errors of semi-major axis $\left(\sigma_{a}\right)$ and eccentricity $\left(\sigma_{e}\right)$ are considered as $100 \mathrm{~km}$ and 0.1 . Errors of inclination $\left(\sigma_{i}\right)$, right ascension of ascending node $\left(\sigma_{\Omega}\right)$ and argument of perigee are all considered as $1 \mathrm{deg}$. Also, ten percent of least required fuel mass is considered as the acceptable excessive fuel mass. i.e. $\sigma_{m}=0.1 \times m_{i m}$.

Intersection of initial and final orbits occurs at $\vec{r}_{0}=-17914 \hat{\mathrm{i}}+2782 \hat{\mathrm{j}}+6153 \hat{k} \mathrm{~km}$. This radius is related to true anomalies of $70^{\circ}$ and $76^{\circ}$ in initial orbit and final orbits respectively.

Since this transfer is a 3D orbital maneuver, all orbital parameters are considered in calculating $J_{e}$. Setting $\lambda=1$ yields to have a unique weighting coefficient for accuracy $\left(J_{e}\right)$ and mass consumption $\left(J_{m}\right)$.

Before starting the optimization problem with thrust vector defined as fully polynomial functions, the gradient of solution domain can be illustrated as a 3D contour by assuming constant thrust direction and magnitude. Setting the thrust magnitude to $11 \mathrm{kN}$ for a spacecraft with payload mass of $600 \mathrm{~kg}$ results the five cost function terms in equation (4) as two constant thrust directions $(\alpha, \beta)$ as depicted in Figure 4. 

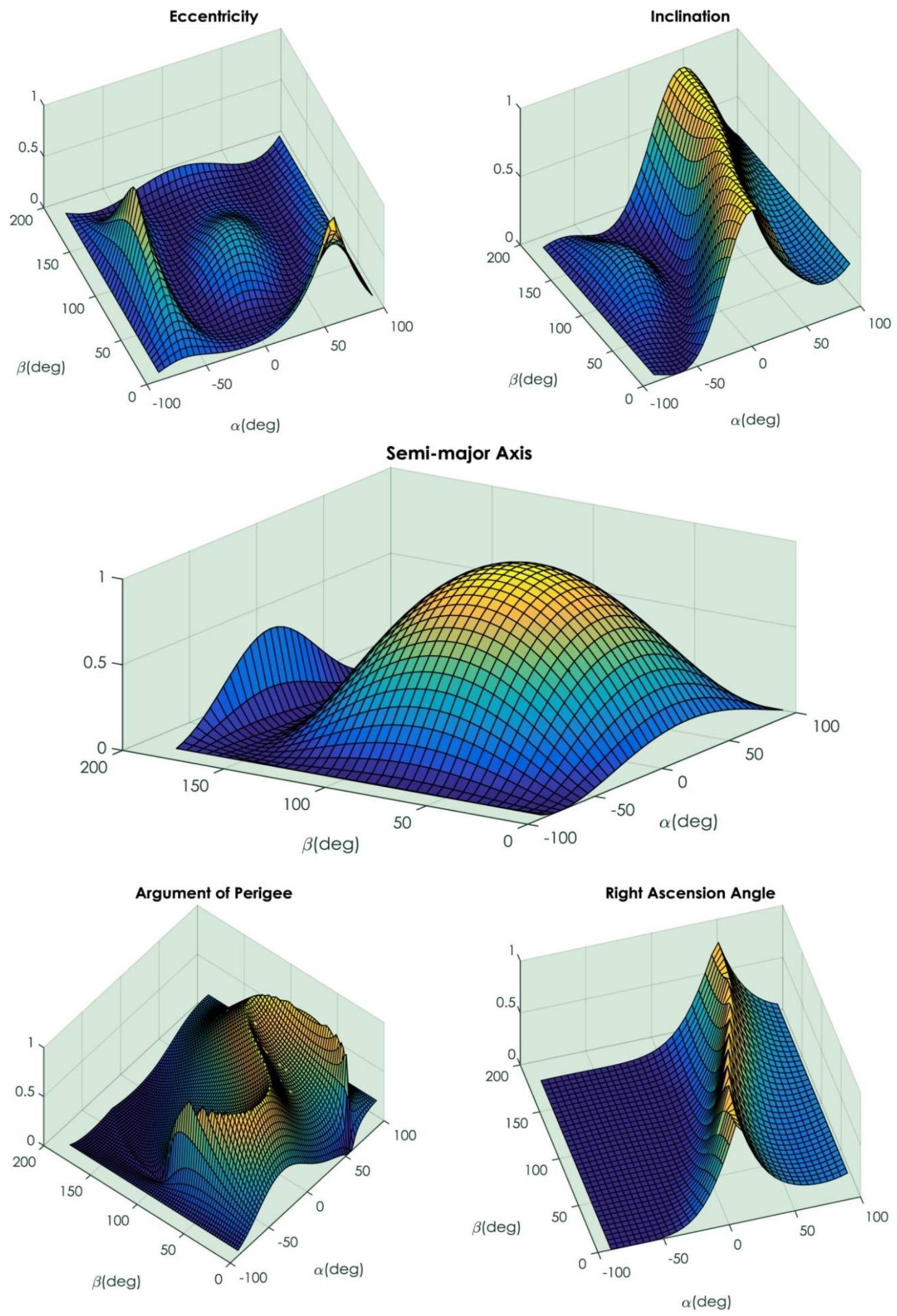

Figure 4. Variation of orbital elements regarding constant thrust direction 
The value of each cost function is scaled in 0 to 1 . As shown in Figure 4, the gradient of solution domain consists of several local minimum points which clearly prove the inapplicability of gradient-based techniques.

Back at the main process, the optimization is carried out using ICA with the following parameters.

Table 2. Parameters, boundaries and stopping criteria in ICA

\begin{tabular}{cc}
\hline Parameter & Value \\
\hline Number of initial countries & 500 \\
\hline Number of initial imperialist & 20 \\
\hline Maximum number of decades & 100 \\
\hline Revolution rate & 0.4 \\
\hline Assimilation coefficient & 1.8 \\
\hline Assimilation angle coefficient & 0.7 \\
\hline Overall scale factor & 1 \\
\hline Stopping criteria & 1 \\
\hline & $-90^{\circ}<\alpha_{c}<+90^{\circ}$ \\
\cline { 2 - 2 } Thrust vector & $R_{\alpha}=30^{\circ}$ \\
\cline { 2 - 2 } shaping boundaries \\
and parameters & $-180^{\circ}<\beta_{c}<+180^{\circ}$ \\
\cline { 2 - 2 } & $R_{\beta}=30^{\circ}$ \\
\cline { 2 - 2 } & $0<T_{c}<20 \mathrm{kN}$ \\
\hline Initial true anomaly & $R_{T}=1000 \mathrm{~N}$ \\
\hline Burn time & $65^{\circ}<\theta_{0}<75^{\circ}$ \\
\hline
\end{tabular}

In this case, a total of 50 points is adopted for each thrust direction angles and a total of 20 points for the thrust magnitude which results in the optimization problem with 125 input variables.

The optimization is conducted using the proposed strategy based on using ICA. Solution converged after 24 iterations. The following solutions are achieved as the optimal thrust vector for the space mission.

$$
\begin{gathered}
T(t)=10.4971+\left(3.5630 \times 10^{-2}\right) \times t \\
+\left(-3.1268 \times 10^{-4}\right) \times t^{2} \\
\alpha(t)=-32.5982+\left(-5.5858 \times 10^{-1}\right) \times t \\
+\left(6.4375 \times 10^{-2}\right) \times t^{2}+\left(-1.5122 \times 10^{-3}\right) \times t^{3} \\
+\left(1.2995 \times 10^{-5}\right) \times t^{4}+\left(-3.7572 \times 10^{-8}\right) \times t^{5}
\end{gathered}
$$

$$
\begin{aligned}
& \beta(t)=166.1909+\left(5.9792 \times 10^{-1}\right) \times t \\
& +\left(-6.7465 \times 10^{-2}\right) \times t^{2}+\left(1.6777 \times 10^{-3}\right) \times t^{3} \\
& +\left(-1.4897 \times 10^{-5}\right) \times t^{4}+\left(4.3545 \times 10^{-8}\right) \times t^{5}
\end{aligned}
$$

As mentioned previously, different polynomial degrees are considered for direction $(n=5)$ and magnitude $(n=2)$ because of different allowable behavior of these parameters. These variations are illustrated as below:

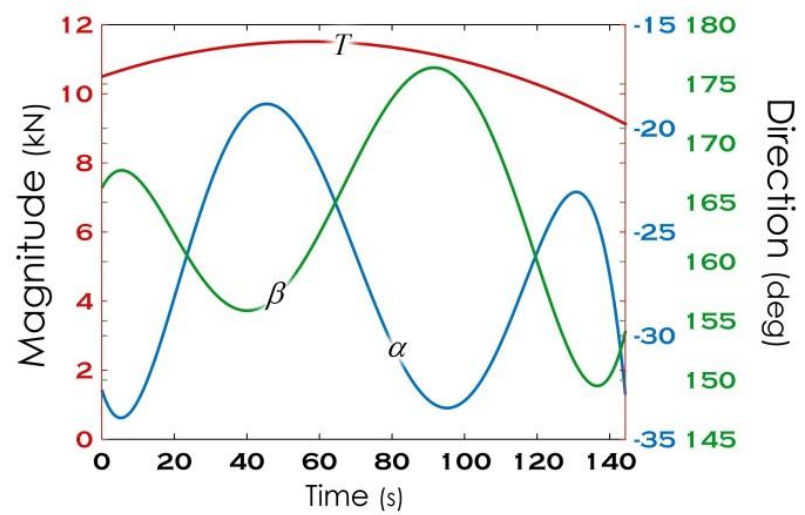

Figure 5. Optimal thrust vector magnitude and direction angles

Regarding Figure 5, it can be seen that the direction angles fairly satisfy the variation margin of $\pm 30^{\circ}$ while employing a cubic thrust magnitude variation. Changes of orbital elements within this transfer are depicted in Figure 6 and Figure 7.

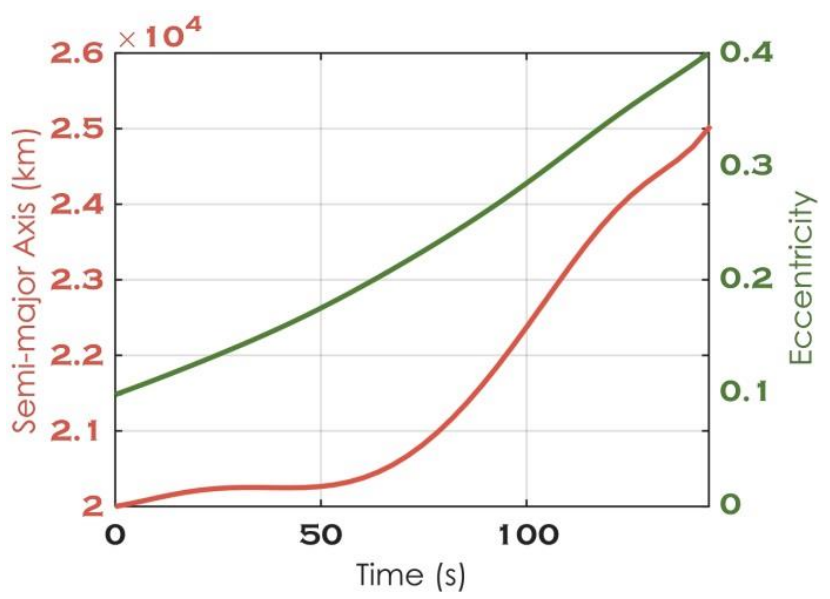

Figure 6. Semi-major axis and eccentricity within the orbit transfer 


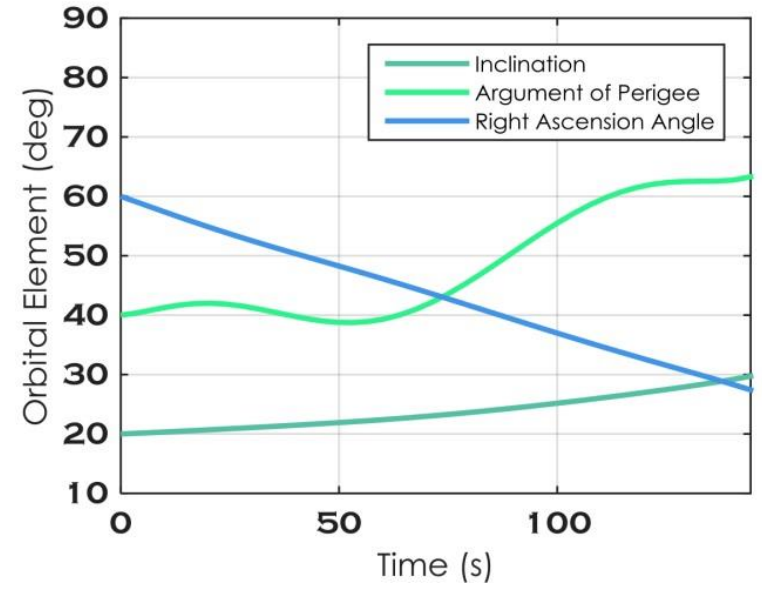

Figure 7. Inclination, argument of perigee and right ascension angle within the orbit transfer

Regarding Figure 6 and 7, the values of semi-major axis and eccentricity at the end of transfer are 2510.32 and 0.4018 respectively. Also, inclination, argument of perigee and right ascension angle are 29.71, 63.28 and 27.41 respectively. It can be seen that the orbital elements reach the desired values with acceptable accuracies defined as mission objective.

The obtained results are based on using a propulsion system which is able to produce cubic thrust profile as illustrated in Figure 5. However, other type of thrust profile can be considered for the space mission based on different propulsion systems. As mentioned previously, the mathematical modeling of thrust magnitude enables the use of different thrust vector behavior for a space mission. Besides neutral variation, regressive and progressive variation is considered for current mission. Clearly the mathematical modeling of thrust magnitude will be more simplistic in this case, leading to use only two points in an acceptable range of thrust magnitude which is considered as $5 \mathrm{kN}$ in this research. The optimal regressive and progressive thrust profiles regarding this approach are depicted in Figure 8.

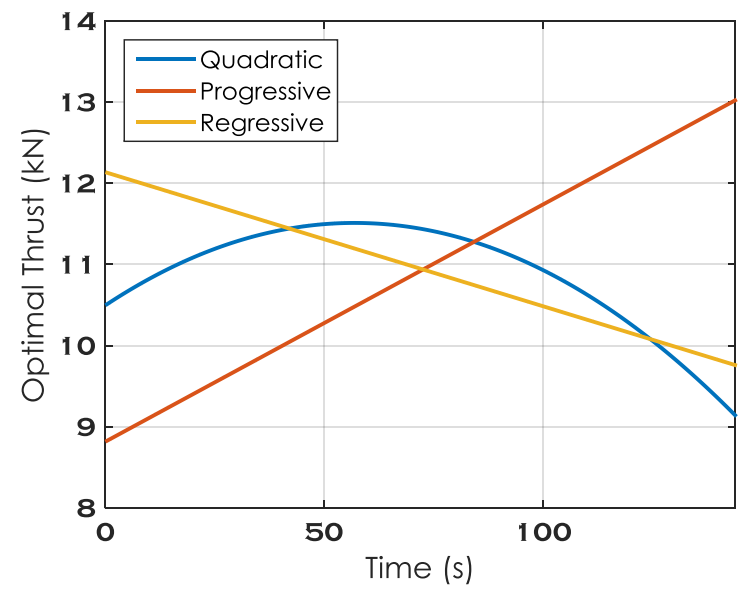

Figure 8. Optimal thrust profiles
As shown in Figure 8, the optimal thrust profiles are generated within the desired margin. The obtained cost functions are shown in Table 3.

Table 3. Characteristics of optimal thrust profiles

\begin{tabular}{c|c|c|c} 
Thrust profile & Quadratic & Progressive & Regressive \\
\hline $\begin{array}{c}\text { Fuel Mass } \\
\begin{array}{c}\text { Maximum } \\
\text { Thrust } \\
\text { Minimum } \\
\text { Thrust }\end{array}\end{array}$ & 535.04 & 536.15 & 537.27 \\
$\begin{array}{c}\text { Cost function } \\
\text { Th }\end{array}$ & $0.127 \mathrm{kN}$ & $13.030 \mathrm{kN}$ & $12.137 \mathrm{kN}$ \\
\hline
\end{tabular}

This table shows the practicality of the proposed approach in finding optimal transfer trajectory regarding different thrust profile behavior.

Because of the finite time required for computation, optimization algorithms with fast convergence time are needed in such systems. Figure 9 compares the convergence process of the presented method and GA.

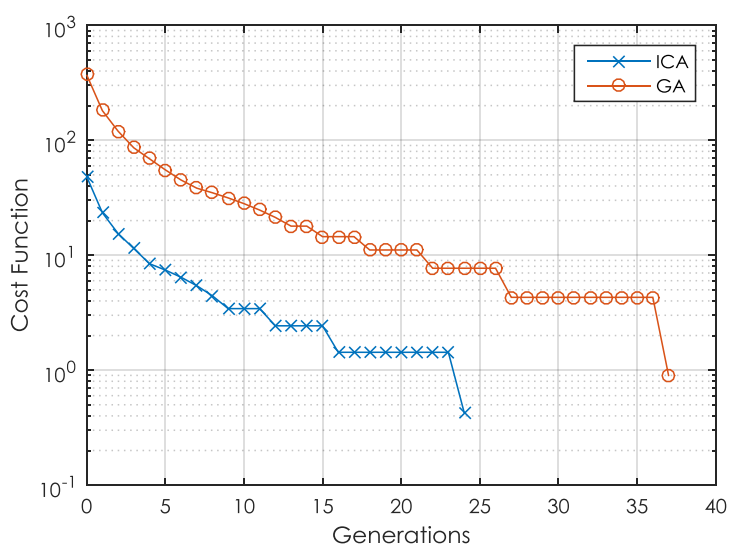

Figure 9. Convergence of the optimization algorithms (ICA, GA)

From Figure 9, it can be seen that the solution converges after 24 generations using ICA, while GA needs 37 generations to reach the optimal solution.

Also, the minor cost functions $J_{e}$ and $J_{m}$ which represent the transfer accuracy and fuel consumption respectively are depicted in Figure 10 for ICA.

It can be observed that the algorithm simultaneously tries to satisfy both objectives while marching through the optimal solution. This means that the selected weighting coefficients for each objective and the orbital parameters are fairly taken into account while using this optimization algorithm. 


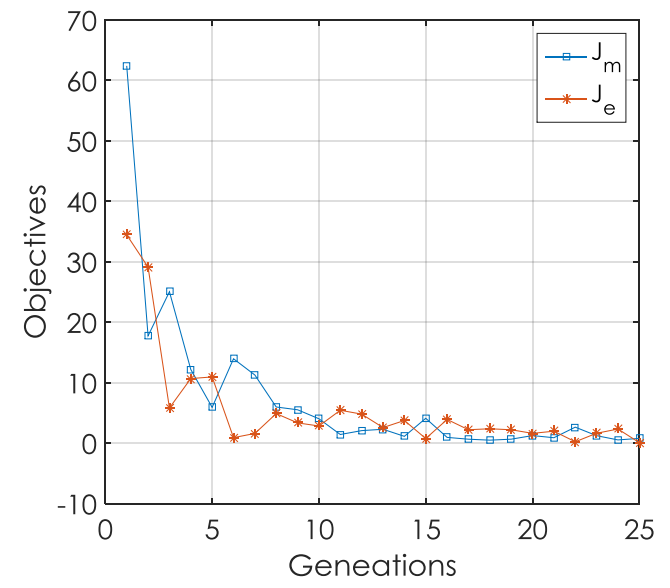

Figure 10. variation of mission objectives within the optimization process (ICA, GA)

By changing the value of the overall weighting coefficients, different multi-objective solutions can be obtained. Pareto frontier is depicted in Figure 11 for current mission.

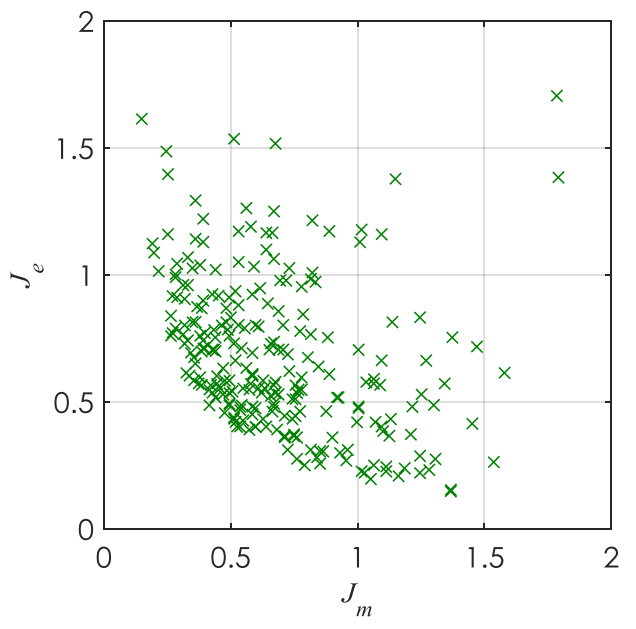

Figure 11. Feasible points of Pareto frontier in trajectory optimization

According to the data provided by Figure 11, it is clear that the physical programming has successfully generated the Pareto-solution front.

Variation of thrust vector direction is depicted in Figure 12.

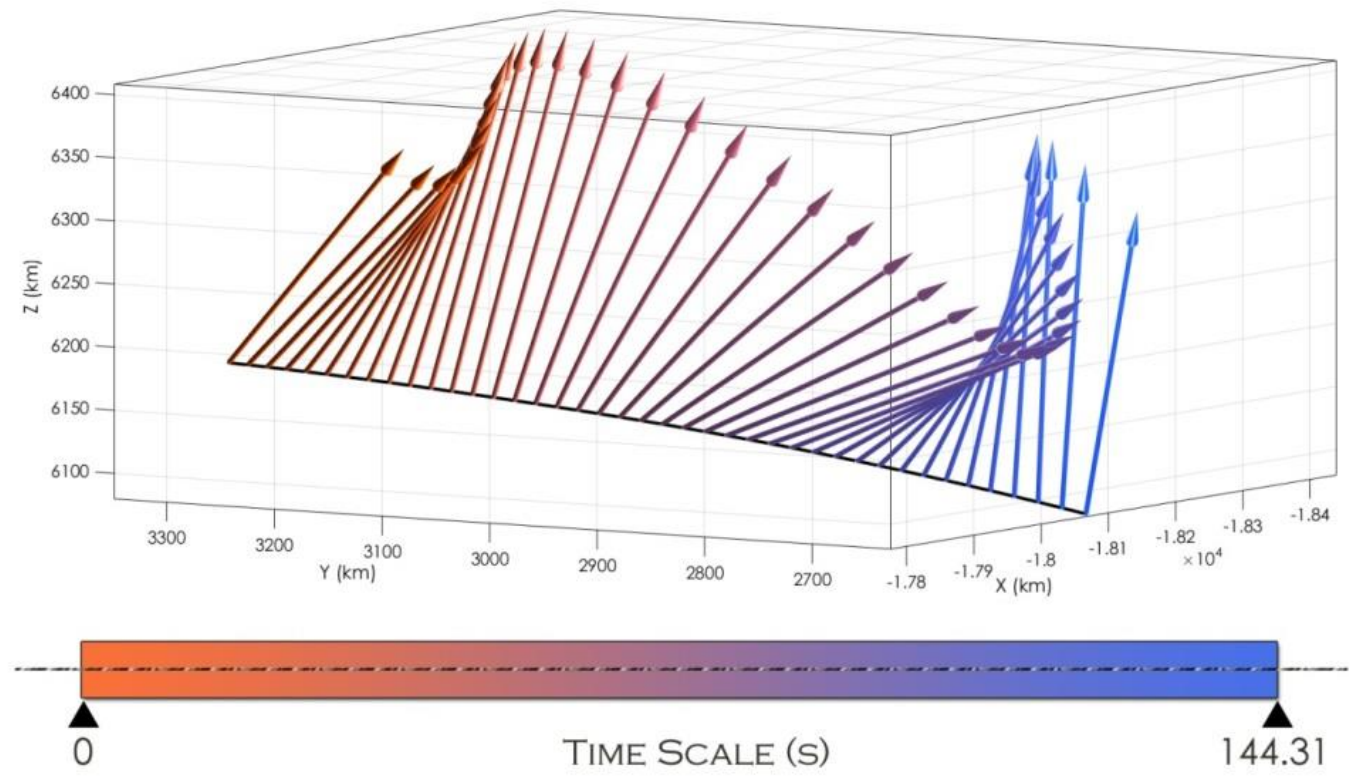

Figure 12. Variation of thrust vector in orbit transfer

There is an obvious relationship between the fuel mass and the transfer accuracy as shown in Figure 11. The less fuel mass dedicated to the spacecraft would result in worse transfer accuracy since the orbital parameters won't reach the desired values. As the fuel mass increases, the transfer will become more optimal. Lastly, the choice of the desired optimal solution depends on the acceptable accuracy along with allowable fuel mass for the space mission.
Simultaneous comparison of Figure 12 and Figure 5 shows that the optimal solution contains noticeable variations for thrust direction angles at special time steps of orbit transfer resulting curved burn arc in which the variation of thrust direction angles are high. 


\section{CONCLUSION AND DISCUSSION}

In this paper, Imperialist Competitive Algorithm was successfully combined with the direct approach and used to solve a high-thrust trajectory optimization problem.

ICA uses the concepts of imperialism and imperialistic competition process as a source of inspiration. ICA starts with an initial population consisting of countries (individuals in other evolutionary algorithms) which are divided in two groups. The ones with the best objective function values are selected to be the imperialists, whereas the remaining ones are their colonies. The colonies are then shared among the imperialists according to each imperialist's power (objective function value). The more powerful an imperialist is, the more colonies it will possess. In the language of ICA an imperialist with his colonies forms an empire.

Polynomials are used as the mathematical modeling of thrust vector magnitude and direction angles. Modifications are made on the basic employment of polynomials to deal with the constrained optimization problem and the premature convergence of the ICA. Considering the computational efficiency of the algorithm, three various thrust profiles were modeled and tested numerically by finding the solution of a fixed-time fuel-optimal high-thrust transfer problem.

The mathematical modeling of inputs has the global search behavior of the ICA as well as the relatively large radius of convergence of the direct technique. These features enable it to obtain a complex high-thrust trajectory with many thrust and coast segments.

It can be furthermore observed that during imperialistic competition the most powerful empires tend to increase their power, while weaker ones tend to collapse. These two mechanisms lead the algorithm to gradually converge into a single empire, in which the imperialist and all the colonies tend to have the same culture.

Combining the ICA and other heuristic optimization algorithms with the indirect approach will be a direction for further research because the indirect approach can provide the high accurate solution for optimal control problem. Certainly, reducing the sensitivity of the indirect approach will be a challenging problem in the research.

\section{APPENDIX}

Obtaining orbital elements from the state vector can be represented in a few steps [23-28]. Having the state vectors $\overrightarrow{\dot{r}}$ and $\overrightarrow{\dot{v}}$, the magnitude of radius and velocity can be calculated as:

$$
r=\sqrt{\vec{r} \cdot \vec{r}}
$$

$$
v=\sqrt{\vec{v} \cdot \vec{v}}
$$

The magnitude of radial velocity is obtained as:

$$
v_{r}=\frac{\vec{r} \cdot \vec{v}}{r}
$$

if $v_{r}>0$, the spacecraft is flying away from perigee. If $v_{r}<0$, it is flying toward perigee. The specific angular momentum vector and its magnitude are calculated as:

$$
\begin{aligned}
\vec{h} & =\vec{r} \times \vec{v} \\
h & =\sqrt{\vec{h} \cdot \vec{h}}
\end{aligned}
$$

Inclination $(i)$ is achieved as:

$$
i=\cos ^{-1}\left(\frac{h_{z}}{h}\right)
$$

Node line vector $(\vec{N})$ and its magnitude are calculated as:

$$
\begin{gathered}
\vec{N}=\hat{k} \times \vec{h} \\
N=\sqrt{\vec{N} \cdot \vec{N}}
\end{gathered}
$$

Right ascension of the ascending node is calculated as:

$$
\Omega= \begin{cases}\cos ^{-1}\left(\frac{N_{x}}{N}\right) & \left(N_{y} \geq 0\right) \\ 360^{\circ}-\cos ^{-1}\left(\frac{N_{x}}{N}\right) & \left(N_{y}<0\right)\end{cases}
$$

Eccentricity vector and its magnitude are calculated as:

$$
\begin{gathered}
N=\sqrt{\vec{N} \cdot \vec{N}} \\
e=\sqrt{\vec{e} \cdot \vec{e}}
\end{gathered}
$$

Having the eccentricity, semi-major axis (a) can be obtained as:

$$
a=\frac{h^{2}}{\mu\left|1-e^{2}\right|}
$$

Argument of perigee is calculated as: 


$$
\omega= \begin{cases}\cos ^{-1}\left(\frac{\vec{N} \cdot \vec{e}}{N e}\right) & \left(e_{z} \geq 0\right) \\ 360^{\circ}-\cos ^{-1}\left(\frac{\vec{N} \cdot \vec{e}}{N e}\right) & \left(e_{z}<0\right)\end{cases}
$$

\section{ACKNOWLEDGEMENTS}

This research is supported by the Basque Government through the ELKARTEK and BERC 2014-2017 programs and by Spanish Ministry of Economy and Competitiveness MINECO: BCAM Severo Ochoa excellence accreditation SEV-2013-0323.

\section{REFERENCES}

[1]. E. Taheri and O. Abdelkhalik, "Fast Initial Trajectory Design for Low-Thrust RestrictedThree-Body Problems", Journal of Guidance, Control, and Dynamics, vol. 38, no. 11, pp. 2146-2160, 2015.

[2]. S. Li, Y. Zhu and Y. Wang, "Rapid design and optimization of low-thrust rendezvous / interception trajectory for asteroid deflection missions", Advances in Space Research, vol. 53, no. 4, pp. 696-707, 2014.

[3]. M. Ceriotti and J. Sanchez, "Control of asteroid retrieval trajectories to libration point orbits", Acta Astronautica, vol. 126, pp. 342-353, 2016.

[4]. M. Dileep, S. Kamath and V. Nair, "Particle Swarm Optimization Applied to Ascent Phase Launch Vehicle Trajectory Optimization Problem", Procedia Computer Science, vol. 54, pp. 516-522, 2015.

[5]. F. Alonso Zotes and M. Santos Peñas, "Particle swarm optimisation of interplanetary trajectories from Earth to Jupiter and Saturn", Engineering Applications of Artificial Intelligence, vol. 25, no. 1, pp. 189-199, 2012.

[6]. S. Hosseini and A. Al Khaled, "A survey on the Imperialist Competitive Algorithm metaheuristic: Implementation in engineering domain and directions for future research", Applied Soft Computing, vol. 24, pp. 1078-1094, 2014.

[7]. H. Shabani, B. Vahidi and M. Ebrahimpour, "A robust PID controller based on imperialist competitive algorithm for load-frequency control of power systems", ISA Transactions, vol. 52, no. 1, pp. 88-95, 2013.

[8]. M. Anjomshoa, A. Mahani and S. Sadeghifard, "A new automated design and optimization method of CMOS logic circuits based on Modified Imperialistic Competitive Algorithm", Applied Soft Computing, vol. 21, pp. 423-432, 2014.

[9]. M. Ghasemi, S. Ghavidel, M. Ghanbarian, H. Massrur and M. Gharibzadeh, "Application of imperialist competitive algorithm with its modified techniques for multi-objective optimal power flow problem: A comparative study", Information Sciences, vol. 281, pp. 225-247, 2014.

[10]. S. Jamali, S. Malektaji and M. Analoui, "An imperialist competitive algorithm for virtual machine placement in cloud computing", Journal of Experimental \& Theoretical Artificial Intelligence, pp. 1-22, 2016.

[11]. I. Shafieenejad, A. Novinzadeh and V. Molazadeh, "Introducing a novel algorithm for minimum-time low-thrust orbital transfers with free initial condition", Proceedings of the Institution of Mechanical Engineers, Part G: Journal of Aerospace Engineering, vol. 229, no. 2, pp. 333-351, 2014.

[12]. H. Curtis, Orbital mechanics for engineering students, 3rd ed. Elsevier, 2014.

[13]. A. Shirazi, "Trajectory optimization of spacecraft high-thrust orbit transfer using a modified evolutionary algorithm", Engineering Optimization, vol. 48, no. 10, pp. 1639-1657, 2015.

[14]. A. Shirazi and A. Mazinan, "Mathematical modeling of spacecraft guidance and control system in 3D space orbit transfer mission", Comp. Appl. Math., 2015.

[15]. Z. Wang, Y. Jia, L. Jin and J. Duan, "Thrust vector control of upper stage with a gimbaled thruster during orbit transfer", Acta Astronautica, vol. 127, pp. 359-366, 2016.

[16]. J. Orr and N. Slegers, "High-Efficiency Thrust Vector Control Allocation", Journal of Guidance, Control, and Dynamics, vol. 37, no. 2, pp. 374382, 2014.

[17]. S. Hu, J. Chen, G. Wu, Q. Xu, H. Liu and Y. Hua, "Burning Behavior of Solid Propellants at High Pressure", Combustion Science and Technology, vol. 186, no. 12, pp. 1858-1888, 2014.

[18]. H. Tian, X. Li, P. Zeng, N. Yu and G. Cai, "Numerical and experimental studies of the hybrid rocket motor with multi-port fuel grain", Acta Astronautica, vol. 96, pp. 261-268, 2014.

[19]. E. Song, S. Cho, C. Park, W. Roh, M. Joh, "Stability analysis of explicit guidance laws for space launch vehicles with varying thrust integrals", Advances in Space Research, vol. 48, no 1, pp. 133-145, 2011

[20]. P. Sforza, Theory of aerospace propulsion. Waltham, MA: Academic Press, 2012.

[21]. M. Dossary, H. Nasrabadi, "Well placement optimization using imperialist competitive algorithm", Journal of Petroleum Science and Engineering, vol. 147, pp. 237-248, 2016

[22]. Atashpaz-Gargari, E., and C. Lucas. "Imperialist Competitive Algorithm: An Algorithm for Optimization Inspired by 
Imperialistic Competition.” Evolutionary Computation, Congress On, 2007

[23]. G. Hintz, Orbital mechanics and astrodynamics, Springer, 2015

[24]. M. Capderou, Satellites Orbits and Missions, Springer, 2005

[25]. Bate, Roger, Donald D Mueller, and Jerry E White. Fundamentals Of Astrodynamics. 1st ed. New York: Dover Publications, 1971

[26]. D. Vallado and W. McClain, Fundamentals of astrodynamics and applications, 1st ed. New York: Springer, 2007

[27]. V. Chobotov, Orbital mechanics, 1st ed. Reston, Va.: American Institute of Aeronautics and Astronautics, 2002.

[28]. R. Madonna, Orbital mechanics, 1st ed. Malabar, Fla.: Krieger Pub. Co., 1997.

\section{BIOGRAPHY}

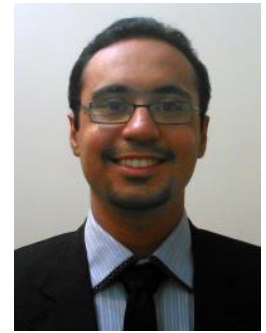

Abolfazl Shirazi is a Ph.D Fellow at BCAM - Basque Center for Applied Mathematics. He received his B.Sc. and M.Sc. degrees in Aerospace Engineering in 2010 and 2012 respectively. The focus of his research includes dynamics and control of spacecraft as well as orbital mechanics and optimization. In his doctoral program, he works on the development of evolutionary algorithms and innovative optimization approaches for spacecraft trajectory design. 